D. Mark (1989) Cognitive Image-Schemata for Geographic Information: Relations to User Views and GIS Interfaces. in: GIS/LIS'89, Orlando, Florida, November 1989 , pp. 551-560.

D. Mark and A. Frank (1996) Experiential and Formal Models of Geographic Space Environment and Planning $B$ 23:3-24.

G. McCalla, L. Reid, and P. Schneider (1982) Plan Creation, Plan Execution, and Knowledge Acquisition in a Dynamic Microworld. International Journal of ManMachine Studies 16(89-112).

D. McDermott and E. Davis (1984) Planning Routes through Uncertain Territory. Artificial Intelligence 22: 107-156.

M. O'Neill (1991a) Effects of signage and floor plan configuration on wayfinding accuracy. Environment and Behavior 23: 553-574.

M. O'Neill (1991b) Evaluation of a conceptual model of architectural legibility. Environment and Behavior 23: 259-284.

J. Piaget and B. Inhelder (1967) The Child's Conception of Space. Norton, New York.

M. Rodríguez and M. Egenhofer (1997) Image-Schemata-Based Spatial Inferences: The Container-Surface Algebra. Department of Spatial Information Science and Engineering, University of Maine, USA, Technical Report.

A. Seidel (1982) Way-Finding in Public Spaces: The Dallas/Fort Worth, USA Airport. in: 20th International Congress of Applied Psychology, Edinburgh, Scotland.

A. Siegel and S. White (1975) The development of spatial representations of largescale environments. in: H. Reese (Ed.), Advances in child development and behavior. 10, Academic Press, New York.

W. Tobler (1976) The geometry of mental maps. in: R. Golledge and G. Rushton (Eds.), Spatial choice and spatial behavior. pp. 69-81, Ohio State University Press, Columbus, Ohio.

B. Tversky (1993) Cognitive Maps, Cognitive Collages, and Spatial Mental Model. in: A. Frank and I. Campari (Eds.), Spatial Information Theory: Theoretical Basis for GIS. Lecture Notes in Computer Science 716, pp. 14-24, Springer Verlag, Heidelberg-Berlin.

J. Weisman (1981) Evaluating architectural legibility: Way-finding in the built environment. Environment and Behavior 13: 189-204.

\section{Fiat and Bona Fide Boundaries: Towards an Ontology of Spatially Extended Objects*}

\section{Barry Smith}

Department of Philosophy and Center for Cognitive Science State University of New York at Buffalo, Buffalo, New York (USA)

\section{Achille C. Varzi}

Department of Philosophy

Columbia University, New York, New York (USA)
Abstract - Human cognitive acts are directed towards objects extended in space of a wide range of different types. What follows is a new proposal for bringing order into this typological clutter. The theory of spatially extended objects should make room not only for the objects of physics but also for objects at higher levels, including the objects of geography and of related disciplines. It should leave room for different types of boundaries, including both the bona fide boundaries which we find in the physical world and the fiat (or human-demarcation-induced) boundaries with which much of geography has to deal. Two distinct axiomatic theories of boundaries are accordingly presented, and the need for both is examined in some detail. The resultant dual framework is shown to have application above all for our understanding of issues involving contact, division, and separation, issues which have posed serious difficulties for the ontological theories of boundaries that have been proposed hitherto.

\section{Articulations of Reality}

The focus of our investigations is the region of objects extended in space. Examples of such objects are: John and Mary, the Moon, the island of Malta. Objects are possessed of divisible bulk: they can be divided, in reality or in thought, into spatial parts. But objects do not merely have constituent object-parts. They also have boundaries, which contribute as much to their ontological make-up as do the constituents they comprehend in their interiors. Our basic categorial scheme will thus recognize, in addition to objects, also what we shall call the outer boundaries of objects.

What, now, of inner boundaries, the boundaries of the interior parts of objects? Imagine a perfectly homogeneous object, for example a spherical ball made of some perfectly homogeneous prime matter. There is a sense in which there are no bounda-

* Thanks are due to the National Center for Geographic Information and Analysis which has provided valuable support to Smith in his work on the present project. 
ries to be acknowledged within the interior of such an object, for it seems that the possession by an object of genuine inner boundaries presupposes either some interior spatial discontinuity ${ }^{1}$ or some qualitative heterogeneity (of material constitution, colour, texture, electric charge, etc.) among its parts. Thus there are many genuine twodimensional (sphere- and torus-like) inner boundaries within the interior of John's body in virtue of the qualitative differentiation of this body into organs, cells, etc. It is clear, however, that we do sometimes speak of inner boundaries even in the absence of corresponding physical discontinuities or intrinsic qualitative differentiation. Examples are: the equator, the International Date Line, the North Pole, and so on. Even in relation to the imagined perfectly homogeneous sphere we can talk sensibly of its upper and lower hemispheres, its centre of mass, etc.

Let us call genuine inner boundaries of the first sort bona fide boundaries, inner boundaries of the second sort fiat boundaries. ${ }^{2}$ This distinction applies not solely to inner boundaries but to outer boundaries, too. The surfaces of extended objects such as planets or tennis balls are of the bona fide sort. National borders, by contrast, as well as county- and property-lines and the borders of postal districts, provide examples of outer boundaries of the fiat sort, at least in those cases where, as in the case of Colorado, Wyoming or Utah, they lie skew to any qualitative differentiations or spatial discontinuities (coastlines, rivers) in the underlying territory.

But now, once fiat outer boundaries have been recognized, it becomes clear that the bona fide-fiat opposition can be drawn not merely in relation to boundaries but in relation to objects also. Examples of bona fide objects are: John and Mary, the planet Earth. Examples of fiat objects are: Dade County, the State of Wyoming, Dogger Bank. And there are of course also mixed cases, objects whose boundaries involve fiat and bona fide segments, such as Alaska, the Northern Hemisphere, the North Sea. It is in relation to the fiat and mixed objects in our local geographic environment - above all in relation to parcels of real estate - that one indispensable role of the surveyor in our society is to be understood. Were it the case that only easily recognizable topographical features of this local environment were of significance in our lives, the role of the surveyor would be confined to the mapping of large-scale features of the terrain features inaccessible to unaided perception, which are of importance for navigation, route-planning and the like.

It will suffice, for present purposes, to confine our attentions to those outer boundaries of objects which can properly be conceived as thin extremal slices. Clarity in relation to boundaries of this sort will be an indispensable presupposition of any more general theory. Certainly there are other varieties of boundary phenomena, including those boundaries commonly conceptualized in terms of zones-objects of the types depicted for example in weather maps, dialect atlases, and the like. A full theory of spatially extended objects must of course cover these cases also, and it must cover also those varieties of objects which have shifting borders (coastal borders are of this sort) as well as varieties of objects which exist in spatial reality but which are yet in some sense indeterminate because they are infected to a high degree by the component

${ }^{1}$ See Casati and Varzi 1994, Ch. 6, "The Natural History of Discontinuities".

2 This terminology was first introduced in Smith 1994, 1995a. of human conceptualization. Where, for example, are the borders of the Carribean? Of Downtown Philadelphia'? Of (Greater) Serbia? Questions like these are sometimes of more than academic significance. As Helen Couclelis points out: 'People kill each other over boundaries of both the well-defined and the undefined kind' (1996, p. 55).

\section{Problems of Contact and Separation}

Consider now a fundamental question that arises as soon the ontological status of boundaries is seriously taken into account. A boundary separates two entities or two parts of the same entity, which are then said to be in contact with each other. How is this contact to be explained? Take the case of the boundary separating the sea from the air. Shall we say, following Brentano (1976), that there are here two boundaries (one of air and one of water) which are exactly co-localized? Or shall we rather follow Bolzano (1851) and maintain that contact is only possible between one entity with a surface and another without, so that, if water and air are to be in contact, then one or the other would have to lack a surface?

\subsection{Varieties of Contact}

We take it that denying the possibility of all contact between separate objects would involve too radical a departure from common sense. To be sure, natural language does not distinguish between true topological contact (or connection, as we may also say) and mere physical closeness. Imagine that two bodies, say John and Mary, converge upon each other for a greater or lesser interval of time, for example in shaking hands or kissing. Physically speaking, as we know, a complicated story has to be told in such cases as to what happens in the area of apparent contact of the two bodies, a story in terms of sub-atomic particles whose location and whose belongingness to either one or other of the two bodies are only statistically specifiable: as far as the bona fide outer boundaries of John and Mary are concerned, no genuine topological contact is possible at all. In general, the surfaces of distinct physical bodies cannot be in contact topologically, though the bodies may of course be so close to each other that they appear to be in contact to the naked eye.

This, however, leaves the question open in those cases where the two candidates for contact are not physical objects. At the very least we want to say that every object must be in contact with its complement (i.e., with the entity that results when we imagine this object as having been subtracted from the universe as a whole). But even this is enough to cause problems. If a circle is divided down the middle into black and white regions, to which region does the line of demarcation belong? To which nation does the boundary between Italy and San Marino belong? Is a body at rest or in motion in the instant when it starts to move? ${ }^{3}$ Such puzzles serve together to call into question the realist attitude towards boundaries, which have accordingly been assigned to almost total oblivion in the history of metaphysics.

\footnotetext{
${ }^{3}$ See Galton 1995 and Smith 1997.
} 
More careful reflection, however, allows us to view the above as the conflict of intuitions concerning the question whether talk of boundaries must always involve a distinction between closed and open entities-i.e., a distinction between entities that do and entities that do not include their boundaries among their constituent parts. ${ }^{4} \mathrm{We}$ shall argue that the view according to which boundaries exist and yield an open/closed dichotomy (a view that amounts to an ontology based on ordinary topology) is correct when the relevant varieties of contact involve bona fide boundaries. However, fiat boundaries, and the analogue of contact which they involve, call for a different account which dispenses with the open/closed distinction altogether. In fact we shall argue that a combination of these two positions will be needed by any complete account of the formal ontology of boundaries.

\subsection{The Open/Closed Opposition}

Those who see the puzzles concerning demarcation referred to above as amounting to a threat to a realist attitude toward boundaries rely-more or less explicitly-on an argument that runs like this: (1) Admitting boundaries implies the open/closed distinction. But (2) the open/closed distinction is counterintuitive-it runs against common sense. (Surely, if we cut an object in half, we are nothout boundaries (and without the and another that is not.) Thus (3) we must do without boundaries (and without the open/closed distinction) and regard talk of boundaries as a mere façon de parler abou other things-for example (as on standard mathematical treatments of the continuum) about infinite series. ${ }^{5}$

We can resist this argument in a number of ways. To begin with, admitting boundaries does not by itself imply the open/closed distinction; hence the premiss (1) of the argument is actually false. This will be seen in some detail below, in relation to the theory of fiat boundaries.

Second, the open/closed distinction is not by itself at odds with common sense rather, it seems to fall outside the domain of mesoscopic phenomena in relation to which common sense-and naive geography-can claim authority. Thus premiss (2) is false also. There may indeed be some familiar entities in relation to which the distinction seems reasonable. Holes, for instance, are bounded from the outside: the boundary of a hole is the surface of its material host (Casati and Varzi 1996). ${ }^{6}$

Third, and most important, the main worry about the open/closed distinctionthat if we cut an object in half, one piece will be closed and the other not-is

4 An entity may include parts of its boundary, but not the whole, and thereby qualify as partly closed and partly open. In the following we shall ignore the complications that arise in such cases and speak of partly open objects as being open tout court We shall restore the strict terminology in the formal developments of Section 3.

5 This position is well exemplified in the recent literature by Tony Cohn and his AI group (whose main concern, however, is not ontology but efficient qualitative reasoning). See see e.g. Randell, Cui, and Cohn 1992, esp. pp. 394f.

${ }^{6}$ See also Asher and Vieu 1995 on the idea that material objects have open complements. For other families of examples see Jackendoff 1991 grounded on a model of cutting that we find questionable (Varzi 1997). Topologically, the intuitive feeling that dissecting a solid reveals matter in its interior and "brings to light new surfaces" (Adams 1984, p. 400) is ill-grounded. Rather, the model we should have in mind, if we wish to understand what happens topologically when a process of cutting takes place, is that of a splitting oil drop. The drop grows longer, slowly but gradually. As it grows, the middle part shrinks and gets thinner and thinner. Eventually the right and left portions split, and we have two drops, each with its own complete boundary. A long, continuous process suddenly results in an abrupt topological change. There was one drop; now there are two. There was one surface, and this surface eventually separated into two. (Think also of a soap bubble splitting. Or think of an island gradually dividing into two.) There appears to be something mysterious in this process, but the mystery would disappear on a more complete assessment, which would require a step into the territories of kinematics and surface elasticity (Davis 1993)

This account reduces the problem of cutting to that of separating two spheres that are connected by one tiny point. But does this really solve the problem? For where does this one point belong - to the left sphere or the right one? Our answer is very simple: the point belongs to both-they overlap. Later we shall explain this more satisfactorily in terms of the notion of fiat boundary. But even without the formal details pertaining to the latter notion, our explanation can be anticipated. There is a tendency to see the phenomenon of cutting as an intuitively clear process that a boundary-based topological account is incapable of explaining; we are arguing that this is misguided. In fact, the problem of cutting is a highly technical one, and not an intuitive question at all-it cannot and should not be solved by any innocent pre-theoretical appeal to naive intuitions. There is indeed something deeply problematic about the point of separation, but this is true of every topological change. Consider:

1) Two drops of oil move toward each other until they come into contact (the opposite of separation). There is a topological catastrophe-literally-that now takes place: the topology of the overall configuration is suddenly altered. Two surfaces merge. Two drops become one.

2) You drill a tunnel into the side of a mountain and break through to the other side. Once again we may speak of a topological catastrophe taking place at the termination of such a process; the topology of the object undergoes an abrupt, qualitative change. To make things simpler we may also ignore the complications involved in the process or drilling - a process that involves removal of matter. Just think of a piece of soft plasticine (or a mushy blob) through which you make a perforation by slowly pressing your finger: there then occurs a constant elastic deformation which terminates when your finger-mirabile dictu-breaks through to the other side?

3) You can also bring a hole into existence by different means-for instance by gluing. Imagine for instance that an island starts growing a "finger" somewhere. Suppose the finger continues its growth until it eventually comes round to meet the main

${ }^{7}$ For more on this, see Ch. 6 of Leyton 1992. 
body of the island once again, forming a sort of handle. At the instant that it does so, the topology of the object suddenly changes.

Stories like these all involve something genuinely problematic. And there is nothing wrong with this. Topological mystery is all around us. It marks the point where common sense and naive geography reach the limits of their theoretical competence (Smith 1995a, Egenhofer and Mark 1995).

From the perspective of physical science, in contrast, ordinary physical objects are not continuous and do not have boundaries of the sort countenanced by the common-sense ontology of the world. If the solid bodies of common sense are replaced by intricate systems of subatomic particles, speaking of a body's surface is like talking of the flat top of a fakir's bed of nails. ${ }^{8}$ Surfaces become imaginary entities enveloping clouds of matter at lower levels, and their shape and properties involve the same sort of arbitrariness as is involved whenever any graph is created by smoothing out a set of scattered data. Then the mysterious moments of topology lose all their mystery. You make a tunnel by removing the last molecule or atom. You split two things when you pull apart the last two molecules or atoms in such a way as to create a gulf between them. No mystery is left. But what follows from this? Not that we should give up talk of boundaries (and topological talk) altogether. For even if we wish to stay close to the ontology of the physical sciences, the fact remains that space itself is most naturally regarded as a continuum within which things are free to move. And if topological talk is deemed inadequate with respect to the entities of atomic physics, one still needs it when it comes to the spatial regions occupied by the putative objects of ordinary discourse.

\subsection{The Ownership of Boundaries}

We still have a puzzle, however. Take any object $x$. Does the boundary of $x$ belong to $x$ or to its complement? Does the boundary inherit the qualities-for example, colour qualities- - of $x$ or of its complement? There is no room for topological mystery here. Yet how can we answer these questions without selecting one or other term at random?

Some cases seem clearer than others. Ordinary material objects, for instance, are in unproblematic fashion the owners of their surfaces. Thus, where a complement meets an object of this sort, the object will be closed and the complement open. We may also argue on the other side that immaterial bodies such as holes are not the owners of their boundaries: where a hole meets its material host, the boundary of the hole is the surface of the host. Again this is unproblematic, since the hole is itself a part of the host's open complement. But even such simple cases may give rise to certain puzzles. For consider any hole that is not entirely surrounded by matter-like the mouth of a vulcano or a hollow such as the Grand Canyon. The hole is in contact with the host; but there are also some regions of its boundary-corresponding to the openings of the hole facing up towards the sky-that are not thus in contact. The

${ }^{8}$ The terminology is taken from Simons 1991, p. 91. We do not of course deny that classical mechanics is built on the assumption of continuous, well-bounded bodies and that this assumption still retains its value for many purposes. question then is: Where do we place the boundaries corresponding to those regions? Within the hole? Within the sky? Either choice would seem arbitrary.

Part of the problem here lies in the trade-off between our theory of objects and our theory of boundaries. The latter must explain what it means for two things to be connected, but it does not need to give a full explanation of the underlying metaphysical (or physical) grounds. Thus, whether the boundary between hole and complement belongs to the hole or to the complement-or whether the boundary is of the fiat or bona fide type - is a question which might be answered specifically by the theory of holes, not by a general theory of boundaries.

However this is not the whole story. For consider again the cutting of a solid object. We argued that the cutting does not bring to light a new surface. But, of course, we can at least conceptualize a new, potential surface right there where the cut would be. As we have seen, our ordinary description of the world very often and quite naturally makes reference to fiat boundaries of this sort, even in the absence of any corresponding discontinuity or qualitative heterogeneity among the parts of the objects involved. And here the open/closed distinction seems to face a real problem. For in the case of fiat boundaries there is no fact of the matter that can support their belonging to one or the other of two adjacent entities. Hence we cannot defer the solution to a theory of the extended entities at issue. The boundary demarcating the upper and lower halves of a sphere of homogeneous stuff is not only hard to assign to either half; it cannot be assigned, no matter what our theory of spheres might look like. And we cannot simply say that it belongs to neither, treating both halves as semi-open entities. The upper and lower hemispheres use up the whole sphere by definition-no boundary can be left as a thin, unowned slice between them.

\subsection{Coincidence of Fiat Boundaries}

It is here that the peculiarity of fiat boundaries comes into play. Fiat boundaries are in a sense potential in that they do not actually separate anything from anything-they do not mark any actual discontinuity. However, it is not that they can come to mark a discontinuity by having their status changed from fiat to bona fide. For fiat boundaries are not the boundaries that would envelop the interior parts with which they are associated in case those parts were brought to light by separating the remainder. The categorial distinction between fiat and bona fide boundaries is absolute. Cutting the Earth in half would not bring the equator to light in such a way that one and the same entity would be transformed from fiat to bona fide status. Rather, it would yield two Earthhalves, each enveloped by a closed connected surface, in such a way that the equator itself is gone forever.

The open/closed account for bona fide boundaries is thus not affected by those demarcation puzzles raised by the possibility of drawing fiat boundaries at will. But how do we account for the ownership of fiat boundaries as such? Which hemisphere does the equator belong to? The answer is that the two hemispheres actually share the equator. The equator belongs to both. Or, more precisely, each hemisphere has its own equator, and the two equators coincide (i.e., have the same spatial location). 
This suggestion draws on Brentano's view, which in fact regards the possibility of coincidence as a distinguishing feature of all boundaries. Brentanian boundaries are located in space-time, but they do not occupy (fill out) space-time; they can therefore be co-located one with another. ${ }^{9}$ This means that for Brentano there is no need for the open/closed opposition, since for him topological connection is due to boundary coincidence. However, we need not embrace this account as a general theory of boundaries. Thus we may not wish to go as far as saying that if a white and a black surface are in contact with each other, then a white and a black line coincide. ${ }^{10}$ For we have seen that the demarcation puzzle is not a problem for the general theory of boundaries when the demarcation is due to a genuine qualitative discontinuity (a bona fide boundary). Rather, we want to regard Brentano's theory as a theory of what goes on when two (potential) parts of an actual entity are separated by fiat. It is when it comes to the notion of contact or adjacency induced by fiat boundaries that coincidence relations become relevant. We can still speak of the equator as a single thing. But, strictly speaking, such a thing is to be recognized as being made up of two perfectly coinciding fiat boundaries bounding the Northern and the Southern hemispheres, respectively. When two soil- or vegetation-types give the appearance of having boundaries which coincide in the region where they come together, this is, from the standpoint we are now defending, appearance only: the phenomenon of coincidence of boundaries is restricted entirely to the fiat realm, and we are confident that close examination of the underlying physics of the soils or plants in question would lend support to this position

\section{Formal Developments}

We thus have two complementary boundary theories. According to the first, more classical theory, genuine contact is only possible between two entities one of which is open and the other closed in the relevant area of contact. The theory of boundary phenomena of the second sort (those involving fiat boundaries) turns on a contrary insight, according to which what is above all characteristic of a continuum is the possibility of a coincidence of boundaries.

The two theories are not completely in disagreement. For instance, both bona fide and fiat boundaries arguably share a fundamental property: they are ontologically parasitic on (i.e., cannot exist in isolation from) their hosts, the entities they bound. This is a common feature that an overall theory of boundary phenomena should emphasize. On the other hand, the two theories yield different notions of contact, and so should be kept distinct. In this final section we shall attend to the task of providing a precise formulation of both theories, starting with their common core and moving then to the two needed supplements.

${ }^{9}$ Brentano's views have been examined by Roderick Chisholm in a number of papers (see e.g. 1984, 1992/3). See also Smith 1997, which provides a detailed formal theory. On the distinction between location and occupation, see Casati and Varzi 1996.

10 See Brentano 1976, p. 41; see also Brentano 1924, pp. 357f.

\subsection{The Common Core}

The fundamental ontological property of boundaries was given a clear formulation by Brentano himself (who in turn elaborated on Aristotle's sketchy remarks in the Physics and the Metaphysics): if something continuous is a mere boundary, then it can never exist except in connection with other boundaries and except as belonging to a continuum of higher dimension (Brentano 1976, Part I). There are, in reality, no isolated points, lines, or surfaces, just as there are no isolated shadows or holes. This must be said of all boundaries, including those which possess no dimension at all, such as spatial points and moments of time and movement: a cutting free from everything that is continuous and extended is for them, too, absolutely impossible.

It is of course impossible to do justice to ideas of this sort without resorting in some way to modal notions. However, we shall attempt in what follows to embed the dependent nature of boundaries into a basic non-modal mereological (more generally, mereotopological) framework.

\subsection{Mereology}

Mereology is the formal theory of part-relations-the relations of part to whole and of part to part within a whole. For simplicity, we shall assume a standard extensional mereological framework constructed around the primitive is a part of, which we symbolize by means of ' $\mathrm{P}$ '. ${ }^{11}$ (Intuitively, we take ' $\mathrm{P}(x, y)$ ' to be true when $x$ is any sort of part of $y$, including an improper part, so that $\mathrm{P}(x, y)$ will be consistent with $x$ 's being identical to $y$.) If we define proper parthood and overlap in the usual way:

$$
\begin{array}{ll}
\mathrm{DP} 1 & \mathrm{PP}(x, y):=\mathrm{P}(x, y) \wedge \neg \mathrm{P}(y, x) \\
\mathrm{DP} 2 & \mathrm{O}(x, y):=\exists z(\mathrm{P}(z, x) \wedge \mathrm{P}(z, y)),
\end{array}
$$

then the axioms for this mereological background can be formulated as follows:12

$$
\begin{array}{ll}
\text { AP1 } & \mathrm{P}(x, x) \\
\text { AP2 } & \mathrm{P}(x, y) \wedge \mathrm{P}(y, x) \rightarrow x=y \\
\text { AP3 } & \mathrm{P}(x, y) \wedge \mathrm{P}(y, z) \rightarrow \mathrm{P}(x, z) \\
\text { AP4 } & \mathrm{PP}(x, y) \rightarrow \exists z(\mathrm{P}(z, y) \wedge \neg \mathrm{O}(z, x)) \\
\text { AP5 } & \exists x(\phi x) \rightarrow \exists y \forall z(\mathrm{O}(y, z) \leftrightarrow \exists x(\phi x \wedge \mathrm{O}(x, z))) .
\end{array}
$$

Thus, parthood is axiomatized as a reflexive, antisymmetric, and transitive relation (i.e., a partial ordering) by AP1-AP3. In addition, AP4 ensures that the result of removing a proper part always leaves a remainder, whereas AP5 guarantees that for every satisfied property or condition $\phi$ (i.e. every condition $\phi$ that yields the value true

${ }^{11}$ For an introduction to classical extensional mereology and its main variants, see Simons 1987. On mereological topology see Smith 1993 and Varzi 1996a.

${ }^{12}$ Here and in the sequel initial universal quantifiers are to be taken as understood and variables are to be conceived as ranging over antifiers are to be taken as understood ary-like. 
for at least one argument) there exists an entity, the sum or fusion, containing all the pers among its parts. ${ }^{13}$ This entity will be denoted by $\sigma x(\phi x)$ and is defined contextually as follows:

$$
\text { DP3 } \sigma x(\phi x):=\mathfrak{l} y \forall z(\mathrm{O}(y, z) \leftrightarrow \exists x(\phi x \wedge \mathrm{O}(x, z))) .
$$

With the help of this operator, we can immediately define a corresponding operator for arbitrary products (of overlapping entities); the product of a class of $\phi$-ers is simply the sum of their common parts:

$$
\text { DP4 } \pi x(\phi x):=\sigma z \forall x(\phi x \rightarrow \mathrm{P}(z, x)) .
$$

Other useful notions are also easily defined. In particular, we shall have use for the following quasi-Boolean operators of sum, product and complement:

$$
\begin{array}{lll}
\text { DP5 } & x+y & :=\sigma z(\mathrm{P}(z, x) \vee \mathrm{P}(z, y)) \\
\text { DP6 } & x \times y & :=\sigma z(\mathrm{P}(z, x) \wedge \mathrm{P}(z, y)) \\
\text { DP7 } & \sim x & :=\sigma z(\neg \mathrm{O}(z, x))
\end{array}
$$

\subsection{The Theory of Bona Fide Boundaries}

Let us now proceed to the formulation of the basic principles for boundaries. We shal begin with the theory of bona fide boundaries, which effectively corresponds to an ontology based on ordinary topology; we shall then move on to the theory of fiat boundaries.

We shall symbolize the primitive boundary relation by ' $\mathrm{B}$ ', reading ' $\mathrm{B}(x, y)$ ' as " $x$ is a (bona fide) boundary for $y$ ". We say "boundary for", rather than of, to avoid a too narrow interpretation of boundaries as maximal boundaries. The notion of a maximal boundary of $x$ is then defined, using AP5, as the sum of all boundaries for $x$ :

$$
\mathrm{DB} 1 \quad \mathrm{~b}(x):=\sigma z(\mathrm{~B}(z, x)) \text {. }
$$

The basic axioms for ' $\mathrm{B}$ ' can now be given as follows:

$$
\begin{array}{ll}
\mathrm{AB} 1 & \mathrm{~B}(x, y) \rightarrow \mathrm{B}(x, \sim y) \\
\mathrm{AB} 2 & \mathrm{~B}(x, y) \wedge \mathrm{B}(y, z) \rightarrow \mathrm{B}(x, z) \\
\mathrm{AB} 3 & \mathrm{P}(z, x) \wedge \mathrm{P}(z, y) \rightarrow(\mathrm{P}(z, \mathrm{~b}(x \times y)) \leftrightarrow \mathrm{P}(z, \mathrm{~b}(x)+\mathrm{b}(y))) .
\end{array}
$$

Equivalently, we could set:

$$
\begin{array}{ll}
\text { AB1 }^{\prime} & \mathrm{b}(x)=\mathrm{b}(\sim x) \\
\text { AB2 }^{\prime} & \mathrm{b}(\mathrm{b}(x))=\mathrm{b}(x) \\
\text { AB3' }^{\prime} & \mathrm{b}(x \times y)+\mathrm{b}(x+y)=\mathrm{b}(x)+\mathrm{b}(y) .
\end{array}
$$

These correspond to the standard axioms for topological boundaries. In fact, if we define the operator for topological closure in the obvious way, as always yielding the sum of an entity with its maximal boundary:
DB2

$$
\mathrm{c}(x):=x+\mathrm{b}(x),
$$

then $\mathrm{AB} 1-\mathrm{AB} 3\left(\mathrm{AB} 1^{\prime}-\mathrm{AB} 3^{\prime}\right)$ are easily seen to be tantamount to (the mereologized versions of) the familiar Kuratowski axioms:

$$
\begin{array}{ll}
\text { TB1 } & \mathrm{P}(x, \mathrm{c}(x)) \\
\text { TB2 } & \mathrm{P}(\mathrm{c}(\mathrm{c}(x)), \mathrm{c}(x)) \\
\text { TB3 } & \mathrm{c}(x+y)=\mathrm{c}(x)+\mathrm{c}(y) .
\end{array}
$$

We thus have a straightforward reformulation of much of standard topology based on mereology instead of set theory. In particular, AB1 tells us that bona fide boundaries are always symmetrical, in the sense that every bona fide boundary of an entity is also a boundary of the entity's complement. This allows us to define the relation of connection as follows:

$$
\text { DB3 } \mathrm{C}(x, y):=\mathrm{O}(\mathrm{c}(x), y) \vee \mathrm{O}(\mathrm{c}(y), x) .
$$

Accordingly, if we define adjacency as external connection, i.e., connection without overlap:

$$
\text { DB4 } \mathrm{A}(x, y):=\mathrm{C}(x, y) \wedge \neg \mathrm{O}(x, y),
$$

and if we define closed and open entities in the obvious way:

$$
\begin{array}{ll}
\text { DB5 } & \mathrm{Cl}(x):=\forall z(\mathrm{~B}(z, x) \rightarrow \mathrm{P}(z, x)) \\
\text { DB6 } & \mathrm{Op}(x):=\forall z(\mathrm{~B}(z, x) \rightarrow \mathrm{P}(z, \sim x)),
\end{array}
$$

then we can immediately infer from the above that two entities can be adjacent only if they are not both closed or both open:

$$
\text { TB4 } \mathrm{A}(x, y) \rightarrow(\mathrm{Cl}(x) \rightarrow \neg \mathrm{Cl}(y)) \wedge(\mathrm{Op}(x) \rightarrow \neg \mathrm{Op}(y)) .
$$

Thus, contact between two closed entities is not possible if contact is understood in terms of connection. The contact between John and Mary when they shake hands or kiss is something which falls outside the orbit of topology; it requires a different (e.g. metric) account.

Here is a list of further theorems that can be proved from $A B 1-A B 3$ :

$$
\begin{array}{ll}
\text { TB5 } & \mathrm{C}(x, x) \\
\text { TB6 } & \mathrm{C}(x, y) \rightarrow \mathrm{C}(y, x) \\
\text { TB7 } & \mathrm{B}(x, y) \leftrightarrow \forall z(\mathrm{P}(z, x) \rightarrow \mathrm{B}(z, y)) \\
\text { TB8 } & \mathrm{P}(x, y) \rightarrow \forall z(\mathrm{C}(z, x) \rightarrow \mathrm{C}(z, y)) \\
\text { TB9 } & \forall x(\phi(x) \rightarrow \mathrm{B}(x, y)) \rightarrow \mathrm{B}(\sigma x(\phi x), y) .
\end{array}
$$

The last two of these theorems are especially noteworthy. TB8 highlights the main connection between mereological and topological notions. There are mereotopological theories which also assume the converse of TB8, with the effect of reducing mereology to a part of topology. ${ }^{14}$ By contrast, the possibility that topologically connected 
entities bear no mereological relationship to one another leaves room for a much richer taxonomy of basic mereotopological relations and is therefore preferable (Varzi 1996). As for TB9, this is what justifies our definition DB1 as a definition of the boundary of an object; it shows that boundaries are closed under general sum and therefore under all mereological properties.

At this point, we can get closer to a standard topological structure in various ways by strengthening the set of relevant axioms as desired. In particular, we obtain a structure corresponding to that of a topological space in the usual sense (modulo the mereological rather than set-theoretical basis) by imposing the analogues of the usual closure conditions:

$$
\begin{array}{ll}
\mathrm{AB} 4 & \mathrm{Cl}(x) \wedge \mathrm{Cl}(y) \rightarrow \mathrm{Cl}(x+y) \\
\mathrm{AB} 5 & \forall x(\phi x \rightarrow \mathrm{Cl}(x)) \rightarrow(z=\pi x(\phi x) \rightarrow \mathrm{Cl}(z)),
\end{array}
$$

or, equivalently:

$$
\begin{array}{ll}
\text { AB4' } & \mathrm{Op}(x) \wedge \mathrm{Op}(y) \rightarrow(z=x \times y \rightarrow \mathrm{Op}(z)) \\
\text { AB5' } & \forall x(\phi x \rightarrow \mathrm{Op}(x)) \rightarrow \mathrm{Op}(\sigma x(\phi x)) .
\end{array}
$$

(In $\mathrm{AB} 5$ and $\mathrm{AB} 4$ ', the consequent is in conditional form due to the need to take account of the absence of a null individual.)

This yields a basic reformulation of standard topological ideas which we take to provide an adequate account of the theory of contact yielded by bona fide boundaries We now wish to go further and capture the Aristotelian-Brentanian (and indeed commonsensical) idea that boundaries are "parasitic" entities. This thesis-which stands opposed to the ordinary set-theoretic conception of boundaries as, effectively, sets of points, each one of which can exist though all around it be annihilated - has a number of possible interpretations. One general statement of the thesis would assert that the existence of any boundary is such as to imply the existence of some entity of highe dimension which it bounds. Here, though, we must content ourselves with the formulation of a simpler thesis, to the effect that every boundary is such that we can find an entity which it bounds and which is such as to have interior parts. ${ }^{15}$ To this end, we define the relational predicate of interior parthood:

DB7 $\quad \mathrm{IP}(x, y):=\mathrm{P}(x, y) \wedge \neg \mathrm{O}(x, \mathrm{~b}(y))$;

we define also, for convenience, the predicate is a boundary:

DB8 $\quad \operatorname{Bd}(x):=\exists y \mathrm{~B}(x, y)$.

We can then write:

$$
\mathrm{AB} 6 \quad \mathrm{Bd}(x) \rightarrow \exists z(\mathrm{~B}(x, z) \wedge \exists w \mathrm{IP}(w, z)) .
$$

This is not very strong, however. For as it turns out, we always have $\mathrm{B}(x, y) \rightarrow$ $\mathrm{B}(x, y+w)$ for any arbitrary $w$ that is separate from (i.e., not connected to) the closure

\footnotetext{
15 See Varzi 1997 and Smith 1993, 1997 for the more general formulation.
}

of $y$. Thus $\mathrm{AB} 6$ is satisfied by choosing $w$ open (so that $\operatorname{IP}(w, w)$ ) and setting $z$ equal to the scattered object $x+w$, which trivializes the thesis.

A dependence thesis of the required strength must impose on $z$ in $\mathrm{AB} 6$ at least the additional requirement of being self-connected (being all of a piece). This predicate can be defined in agreement with ordinary usage, according to which an entity is connected if it does not amount to the sum of two disconnected parts:

$$
\text { DB9 } \quad \mathrm{Cn}(x):=\forall y \forall z(x=y+z \rightarrow \mathrm{C}(y, z)) .
$$

We can then amend AB6 to the following thesis affirming, for connected boundaries, the existence of connected wholes which they are the boundaries of:

$$
\mathrm{AB} 6^{\prime} \quad \mathrm{Bd}(x) \wedge \mathrm{Cn}(x) \rightarrow \exists z(\mathrm{Cn}(z) \wedge \mathrm{B}(x, z) \wedge \exists w \mathrm{IP}(w, z)) .
$$

\subsection{The Theory of Fiat Boundaries}

In contrast to the classical topological account, the theory of fiat boundaries leaves room for the possibility that certain boundaries be asymmetrical (so that we might in certain circumstances talk of 'oriented boundaries'). That is, certain boundaries may, on this view, be boundaries only in certain directions and not in others. (Think, for example, of the old boundary between the German Democratic and Federal Republics, which was a boundary in one direction only.)

The Brentanian theory may be formulated by taking as primitive the concept of coincidence. This is to be understood intuitively as a relation that obtains between two boundaries whenever they have exactly the same spatial location. Coincidence, as we shall here use the notion, is also to be understood as the sort of thing that pertains exclusively to boundaries. Extended bodies do not coincide (not even with themselves); nor do they coincide with the spatial regions they occupy.

The basic axioms for coincidence-which we symbolize by ' $\approx$ ' - assert that this relation is symmetric and transitive:

$$
\begin{array}{ll}
\mathrm{A} \approx 1 & x \approx y \rightarrow y \approx x \\
\mathrm{~A} \approx 2 & (x \approx y \wedge y \approx z) \rightarrow x \approx z .
\end{array}
$$

Thus, coincidence is conditionally reflexive:

$$
\mathrm{T} \approx 1 \quad x \approx y \rightarrow x \approx x .
$$

To this we add a further summing principle to the effect that, if two entities coincide with two further entities, then the mereological sum of the first two coincides with the mereological sum of the second two:

$$
\mathrm{A} \approx 3 \quad(x \approx y \wedge w \approx z) \rightarrow x+w \approx y+z .
$$

We may also need to add mixed mereological postulates to guarantee at least weak monotonicity and closure under general sum:

$\mathrm{A} \approx 5 \quad(\exists y(\phi y) \wedge \forall y(\phi y \rightarrow x \approx y)) \rightarrow x \approx \sigma y(\phi y)$. 
Thus, in particular, if $x$ coincides with both $y$ and $z$, then it coincides also with the sum of $y$ and $z$ :

$$
\mathrm{T} \approx 2 \quad(x \approx y \wedge x \approx z) \rightarrow x \approx y+z .
$$

From $A \approx 5$ we can prove also that, for satisfied $\phi$ and $\psi$, concidence of all instances implies coincidence of corresponding mereological sums:

$$
\mathrm{T} \approx 3 \quad \forall x \forall y[(\phi x \wedge \psi y) \rightarrow x \approx y] \rightarrow \sigma x \phi x \approx \sigma y \psi y .
$$
coincide:

Finally, we must adopt an axiom to the effect that parts of self-coincidents self-

$$
\mathrm{A} \approx 6 \quad x \approx x \rightarrow \forall y(\mathrm{PP}(y, x) \rightarrow y \approx y) .
$$

This guarantees that if we now define fiat boundaries as those entities which may enter the coincidence relation: ${ }^{16}$

$$
\mathrm{D} \approx 1 \quad \mathrm{Bd}^{*}(x):=x \approx x,
$$

then every part of a fiat boundary is itself a fiat boundary:

$$
\mathrm{T}=4 \quad \mathrm{Bd}^{*}(x) \wedge \mathrm{P}(z, x) \rightarrow \mathrm{Bd}^{*}(z) .
$$

We are now ready to define Brentanian connection - connection by fiat boundary. The idea is that this form of connection obtains between two adjacent entities whenever their boundaries coincide at least in part. To this end, let us define the relational concept of a fiat boundary of an entity (the fiat analogue of ' $\mathrm{B}$ '). Fiat boundaries are necessarily boundary parts-proper parts of the entities they bound:

$$
\mathrm{D} \approx 2 \quad \mathrm{~B}^{*}(x, y):=\mathrm{Bd}^{*}(x) \wedge \mathrm{PP}(x, y) \cdot{ }^{17}
$$

We can then define the sort of connection that is induced by fiat boundaries as follows:

$$
\mathrm{D} \approx 3 \quad \mathrm{C}^{*}(x, y):=\mathrm{O}(x, y) \vee \exists z \exists w\left(\mathrm{~B}^{*}(z, x) \wedge \mathrm{B}^{*}(w, y) \wedge z \approx w\right) .
$$

Note that the difference between this and the notion of connection defined for bona fide boundaries (DB3) comes to light only in the case of adjacent entities. If the boundary through which adjacent entities are connected is a bona fide boundary, then this by definition bounds one entity from the inside and the other from the outside. (See again TB4, which effectively represents the Bolzanian view of external contact.) If by contrast it is a fiat boundary (i.e., a matter of coincident Brentanian boundaries), then each entity is bounded, as it were, by its own fiat boundary.

At this point, we can formulate the fiat analogues of the fundamental principle of ontological dependence. This is done simply by taking the predicates ' $\mathrm{Bd}$ ', and ' $\mathrm{B}$ '

16 If coincidence were understood broadly so as to hold of other entities besides boundaries, then ' $\mathrm{Bd}^{*}$ ' (or ' $\mathrm{B}$ '' below) would have to be taken as primitive and the conditional corresponding to the left-to-right direction of $\mathrm{D} \approx 1$ would turn into an axiom.

17 There is a form of de re necessity involved in this claim that is lost in the purely extensional definition given in $\mathrm{D} \approx 2$. See Smith 1997 for further details. in place of ' $\mathrm{Bd}$ ' and ' $\mathrm{B}$ ' in the formulations ( $\mathrm{AB} 6$ and $\mathrm{AB} 6$ ') given in the previous section. In particular, the fiat analogue of $\mathrm{AB} 6$ ' becomes:

$$
\mathrm{A} \approx 7 \quad \mathrm{Bd}^{*}(x) \wedge \mathrm{Cn}(x) \rightarrow \exists z\left(\operatorname{Cn}(z) \wedge \mathrm{B}^{*}(x, z) \wedge \exists w \operatorname{IP}(w, z)\right) .
$$

Thus, the theory of fiat boundaries presupposes, in an important sense, the theory of bona fide boundaries (through the notions of interior parthood and connectedness, respectively IP and $\mathrm{Cn}$ ). This is reasonable, for we have seen that bona fide boundaries contribute as much to the ontological make-up of objects as do their extended constituents. By contrast, the demarcations induced by fiat boundaries are not grounded in any intrinsic features of the underlying reality, and correspond only to cognitive phenomena such as those induced by our use and understanding of political maps and cadastral surveys. Fiat boundaries are in this sense superficial only, though their importance for human history is of course far from negligible.

\section{Concluding Remarks}

We hope to have shown that an adequate typology of boundaries must involve an opposition between bona fide (or physical) and fiat (or human-demarcation-induced) boundaries. Many of the problems connected with the common-sense notions of adjacency, contact, separation and division can be resolved in an intuitive way by recognizing this bicategorial nature of boundaries. Bona fide boundaries yield a notion of contact that is effectively modeled by classical topology; the analogue of contact involving fiat boundaries calls for a different account, based on the intuition that such boundaries do not support the open/closed distinction of classical topology. Our suggestion is that it is a combination of these two positions which will provide the correct framework for investigating the ontology of boundaries and of the full range of spatially extended objects which they bound.

\section{References}

Adams, E. W., 1984, 'On the Superficial', Pacific Philosophical Quarterly 65, 386407.

Asher, N., and Vieu, L., 1995, 'Toward a Geometry of Common Sense: A Semantics and a Complete Axiomatization of Mereotopology,' Proceedings of the 14th International Joint Conference on Artificial Intelligence, San Mateo, CA: Morgan Kaufmann, pp. 846-52.

Bolzano, B., 1851, Paradoxien des Unendlichen, hrsg. von F. Prihonsky, Leipzig: Reclam (Eng. trans. by D. A. Steele, Paradoxes of the Infinite, London: Routledge \& Kegan Paul, 1950).

Brentano, F., 1924, Psychologie vom empirischen Standpunkt, 2. Aufgabe, hrsg. von O. Kraus, Leipzig: Meiner (Eng. trans. ed. by L. L. McAlister, Psychology from an Empirical Standpoint, London: Routledge \& Kegan Paul, 1950). 
Brentano, F., 1976, Philosophische Untersuchungen zu Raum, Zeit und Kontinuum, hrsg. von S. Körner und R. M. Chisholm, Hamburg: Meiner (Eng. trans. by B. Smith, Philosophical Investigations on Space, Time and the Continuum, London: Croom Helm, 1988).

Casati, R., and Varzi, A. C., 1994, Holes and Other Superficialities, Cambridge, MA, and London: MIT Press (Bradford Books).

Casati, R., and Varzi, A. C., 1996, 'The Structure of Spatial Localization', Philosophical Studies 82, 205-39.

Chisholm, R. M., 1984, 'Boundaries as Dependent Particulars', Grazer philosophische Studien 10, 87-95.

Chisholm, R. M., 1992/3, 'Spatial Continuity and the Theory of Part and Whole. A Brentano Study', Brentano Studien 4, 11-23.

Clarke, B. L., 1981, 'A Calculus of Individuals Based on "Connection"', Notre Dame Journal of Formal Logic 22, 204-18.

Couclelis, H., 1996, 'Typology of Geographic Entities with Ill-Defined Boundaries,' in P. A. Burrough and A. U. Frank (eds.), Geographic Objects with Indeterminate Boundaries, London and Bristol, PA: Taylor and Francis, pp. 45-56.

Davis, E., 1993, 'The Kinematics of Cutting Solid Objects', Annals of Mathematics and Artificial Intelligence 9, 253-305.

Egenhofer, M., and Mark, D., 1995 'Naive Geography', in A. U. Frank and W. Kuhn (eds.), Spatial Information Theory. A Theoretical Basis for GIS, Berlin/Heidelberg: Springer-Verlag, pp. 1-15.

Galton, A., 1995, 'Towards a Qualitative Theory of Movement', in A. U. Frank and W. Kuhn (eds.), Spatial Information Theory. A Theoretical Basis for GIS, Berlin/Heidelberg: Springer-Verlag, pp. 377-96.

Jackendoff, R., 1991, 'Parts and Boundaries', Cognition 41, 9-45.

Leyton, M., 1992, Symmetry, Causality, Mind, Cambridge, MA, and London: MIT Press.

Randell, D. A., Cui, Z., and Cohn, A. G., 1992, 'An Interval Logic of Space Based on "Connection"', in B. Neumann (ed.), Proceedings of the 10th European Conference on Artificial Intelligence, Chichester: John Wiley \& Sons, pp. 394-98.

Simons, P. M., 1987, Parts. A Study in Ontology, Oxford: Clarendon Press

Simons, P. M., 1991, 'Faces, Boundaries, and Thin Layers', in A. P. Martinich and M. J. White (eds.), Certainty and Surface in Epistemology and Philosophical Method. Essays in Honor of Avrum Stroll, Lewiston/Queenston/Lampeter: Edwin Mellen Press, pp. 87-99.

Smith, B., 1993, 'Ontology and the Logistic Analysis of Reality', in N. Guarino and R. Poli (eds.), Proceedings of the International Workshop on Formal Ontology in Conceptual Analysis and Knowledge Representation, Padova: Ladseb-CNR, pp. 51-68; revised version as 'Mereotopology: A Theory of Parts and Boundaries', Data and Knowledge Engineering 20 (1996), 287-304.

Smith, B., 1994, 'Fiat Objects', in N. Guarino, S. Pribbenow, and L. Vieu (eds.), Parts and Wholes: Conceptual Part-Whole Relations and Formal Mereology. Proceedings of the ECAI94 Workshop, Amsterdam: ECCAI, pp. 15-23.

Smith, B., 1995a, 'Formal Ontology, Common Sense, and Cognitive Science', International Journal of Human Computer Studies 43, 641-67.
Smith, B., 1995b, 'On Drawing Lines on a Map', in A. U. Frank and W. Kuhn (eds.), Spatial Information Theory. A Theoretical Basis for GIS, Berlin/Heidelberg: Springer-Verlag, pp. 475-84.

Smith, B., 1997, 'Boundaries: An Essay in Mereotopology', in L. Hahn (ed.), The Philosophy of Roderick Chisholm, La Salle, IL: Open Court, forthcoming.

Varzi, A. C., 1994, 'On the Boundary Between Mereology and Topology', in R. Casati, B. Smith, and G. White (eds.), Philosophy and the Cognitive Sciences, Vienna: Hölder-Pichler-Tempsky, pp. 423-42.

Varzi, A. C., 1996a, 'Parts, Wholes, and Part-Whole Relations: The Prospects of Mereotopology', Data and Knowledge Engineering 20, 259-86.

Varzi, A. C., 1996b, 'Reasoning about Space: The Hole Story', Logic and Logical Philosophy 4, 3-39.

Varzi, A. C., 1997, 'Boundaries, Continuity, and Contact', Nô̂s 31, 26-58.

Whitehead, A. N., 1929, Process and Reality. An Essay in Cosmology, New York: Macmillan. 